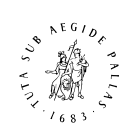

B R I L L

\title{
Aging and Sensitivity to Illusory Target Motion With or Without Secondary Tasks
}

\author{
Alix L. de Dieuleveult ${ }^{1,2,3, *}$, Anne-Marie Brouwer ${ }^{2}$, Petra C. Siemonsma ${ }^{4,5}$, \\ Jan B. F. van $\operatorname{Erp}^{2,3}$ and Eli Brenner ${ }^{6}$ \\ ${ }^{1}$ Predictive Health Technologies, TNO, Leiden, the Netherlands \\ ${ }^{2}$ Perceptual and Cognitive Systems, TNO, Soesterberg, the Netherlands \\ ${ }^{3}$ University of Twente, Enschede, the Netherlands \\ ${ }^{4}$ University of Applied Sciences Leiden, Leiden, the Netherlands \\ ${ }^{5}$ Thim van der Laan, University for Physiotherapy, Nieuwegein, the Netherlands \\ ${ }^{6}$ Vrije Universiteit, Amsterdam, the Netherlands
}

Received 28 February 2017; accepted 7 July 2017

\begin{abstract}
Older individuals seem to find it more difficult to ignore inaccurate sensory cues than younger individuals. We examined whether this could be quantified using an interception task. Twenty healthy young adults (age 18-34) and twenty-four healthy older adults (age 60-82) were asked to tap on discs that were moving downwards on a screen with their finger. Moving the background to the left made the discs appear to move more to the right. Moving the background to the right made them appear to move more to the left. The discs disappeared before the finger reached the screen, so participants had to anticipate how the target would continue to move. We examined how misjudging the disc's motion when the background moves influenced tapping. Participants received veridical feedback about their performance, so their sensitivity to the illusory motion indicates to what extent they could ignore the task-irrelevant visual information. We expected older adults to be more sensitive to the illusion than younger adults. To investigate whether sensorimotor or cognitive load would increase this sensitivity, we also asked participants to do the task while standing on foam or counting tones. Background motion influenced older adults more than younger adults. The secondary tasks did not increase the background's influence. Older adults might be more sensitive to the moving background because they find it more difficult to ignore irrelevant sensory information in general, but they may rely more on vision because they have less reliable proprioceptive and vestibular information.
\end{abstract}

\footnotetext{
* To whom correspondence should be addressed. E-mail: alix.dedieuleveult@tno.nl 


\section{Keywords}

Sensory integration, healthy aging, elderly, dual task, activities of daily living

\section{Introduction}

To be able to live independently, older adults need to properly integrate sensory information from their environment (Lowry et al., 2012) in order to perform both the basic activities of daily living (e.g. bathing, dressing) (Katz, 1963) and the instrumental activities of daily living (e.g., using a phone, shopping) (Lawton and Brody, 1969). It has been shown that aging influences sensory integration (for a review, see de Dieuleveult et al., 2017). Older adults appear to benefit more from multisensory enrichment in their environment than younger adults (Berard et al., 2012; de Dieuleveult et al., 2017; Deshpande and Zhang, 2014; Diederich et al., 2008; Townsend et al., 2006). However, there is also some evidence that older adults have trouble ignoring clearly irrelevant or unreliable sensory information; they use all environmental information even when it is disrupted or non-informative (distractors) (Berard et al., 2012; de Dieuleveult et al., 2017; Eikema et al., 2014; McGovern et al., 2014; Teasdale et al., 1991). In general, when performing various tasks, older adults tend to take more time, to be less accurate, and to be more variable than younger adults (de Dieuleveult et al., 2017; DeLoss et al., 2013; Guerreiro et al., 2014, 2015; Hugenschmidt et al., 2009). The addition of a secondary task tends to decrease task performance more strongly in older adults than in younger adults (Bisson et al., 2014; de Dieuleveult et al., 2017; Mahboobin et al., 2007; Redfern et al., 2001, 2009). It is well known that the age-related deterioration of, for instance, vision (Kavcic et al., 2011; Owsley, 2011), joint mobility (Yeh et al., 2015), muscle force (Cruz-Jentoft et al., 2010), and balance (Teasdale et al., 1991) all decrease performance of the activities of daily living. How changes in sensory integration influence age-related decline in activities of daily living is less well researched.

Vision guides goal-directed reach movements towards moving targets (Brenner and Smeets, 2015; Brouwer et al., 2002, 2003; Kavcic et al., 2011). When judging the target's motion, one could simply rely on the target's retinal slip together with extra-retinal information about eye, head and body movements, but one might be able to improve the precision by assuming that the environment is stable. The relative motion between target and background provides information about target motion that is insensitive to eye or body rotations, with background motion possibly being interpreted as optic flow due to our own motion (Brenner and van den Berg, 1996). However, if the background is moving, relying on such relative motion will lead to systematic errors. When it is clear that the background is moving, and feedback provides 
evidence that relying on motion relative to the background is not justified, it would therefore be beneficial to refrain from relying on relative motion to increase precision. Berard and collaborators found differences between younger and older adults in the ability to down-regulate the influence of such visual information (Berard et al., 2012) in a walking task. Older and younger participants were asked to walk in a straight line in physical space while viewing a 3D scene in a helmet-mounted display unit. Three conditions were presented: one in which the visual scene corresponded with their motion without any visual perturbation, one with no visual input at all, and one with a visual perturbation whereby the focus of expansion of the scene in the visual device gradually rotated to the right or left. They found that younger adults were able to down-regulate the visual information in the perturbed condition, while older adults were not and consequently showed larger deviations in their walking trajectory even though performance was as good as that of young adults when no or only correct visual information was presented. They concluded that old age affects the ability to ignore wrong visual information.

Here we investigate whether this effect generalizes to another paradigm, namely the paradigm of Brouwer et al. (2003). In that study, young participants were asked to hit a disc as quickly and accurately as possible with a rod. The disc was going downward on the screen in one of five different directions. In half of the trials the disc disappeared after $150 \mathrm{~ms}$. In half of those trials the background was static. In the other half the background moved to the left or to the right. The background's movement created an illusion of motion called the Duncker illusion (or induced motion) (Duncker, 1929). The object appears to move differently due to movement in its surrounding (Soechting et al., 2001; Zivotofsky, 2004). Brouwer and collaborators showed that the moving background induced systematic interception errors in accordance with partially relying on the relative motion between the target and the background (systematic errors opposite the direction of background motion). These results are similar to the ones of Berard et al. (2012) in that motion of a large scene induces a deviation of the participants' responses.

As far as we know, the effect of age on the influence of a moving background in interception has not been investigated before. We expect the background motion in our experiment to induce systematic interception errors in the opposite direction to the background motion (as seen in Brouwer et al., 2003) if the background is moving when the target appears. We might expect this effect to be larger for older adults than for younger adults, because, as explained earlier, older adults seem to have trouble ignoring irrelevant visual information (Berard et al., 2012; de Dieuleveult et al., 2017). However, it is well known that older adults have poorer visual object recognition, acuity and contrast sensitivity (Bennett et al., 2007; Owsley, 2011; Pilz et al., 2010). Older adults also have higher motion detection thresholds and they are less 
accurate in discriminating direction and speed (Atchley and Andersen, 1998; Bennett et al., 2007; Conlon et al., 2017; Norman et al., 2003; Pilz et al., 2010; Snowden and Kavanagh, 2006; Trick and Silverman, 1991). Thus, older adults might rely on vision less than younger adults (as seen in Ramkhalawansingh et al., 2017). This makes it not a priori obvious that older adults will respond more to visual information in all situations. Aging is also known to affect various cognitive, somatosensory and muscular systems (Cruz-Jentoft et al., 2010; Vernooij et al., 2016; Yeh et al., 2015), resulting in a loss of behavioral adaptability and a decline in the range of movements that can be made (Newell et al., 2006; Vernooij et al., 2016). It also affects cognitive functions such as attention and memory (Christensen et al., 1994; Glisky, 2007), and older adults have been shown to have difficulties accurately performing multiple tasks at the same time (Bisson et al., 2014; de Dieuleveult et al., 2017; Mahboobin et al., 2007; Redfern et al., 2001, 2009) We therefore expected older adults to also show degraded overall performances in our task, especially when confronted with a secondary balance or cognitive task.

Our main interest was whether older adults would be more susceptible to background motion, and whether any such deficit correlates with performance on well-known clinical tests that we also performed, such as the Modified Clinical Test of Sensory Interaction and Balance (m-CTSIB) and the Short Physical Performance Battery (SPPB), or with their score on the Instrumental Activities of Daily Living scale (Lawton and Brody, 1969). A larger effect of background motion in the presence of a secondary task might reveal compensatory mechanisms that normally help to reduce deficits caused by being unable to ignore irrelevant information.

Finally, we examined susceptibility to a second influence of moving the background: a tendency to temporarily move in the same direction as the background if the background abruptly starts to move when the arm movement is already underway. This effect does not depend on the direction of target motion or whether the target is moving at all (Brenner and Smeets, 1997, 2015; Saijo et al., 2005). In our experiment, such background motion would give rise to a deviation in the opposite direction than that caused by a background that is moving when the target appears. Finding no difference in susceptibility between young and old adults for such background motion would indicate that the difference in performance between older and younger adults in the interception task is specific to judgments of the target's motion.

\section{Material and Methods}

\subsection{Participants}

Twenty-four older adults (60-82 years old, mean age $67 \pm 6.40$ years, nine women) and twenty younger adults (18-34 years old, mean age $25.2 \pm$ 
5.45 years, 11 women) participated in the study. They were recruited via the participant pool of TNO Soesterberg and received a monetary compensation for their participation and travel costs. All participants were naïve with respect to the purpose of the experimental manipulation (background motion) and signed an informed consent form. Participants self-reported being righthanded, having normal or corrected-to-normal vision (participants were asked to put on their glasses or contact lenses if needed) and hearing (hearing was checked by the examiner before doing the experiment by asking the participants whether they heard the low and high tones), and not having been diagnosed as having a vestibular or balance dysfunction, psychiatric symptoms, or musculoskeletal or neurological problems. They self-reported to be in relatively good health during the two weeks prior to the experiment and on the day of the experiment. None of the participants had cognitive impairments as verified by the Mini Mental State Examination (MMSE) used as a screening test with a cut-off score of 24 (Dick et al., 1984). The score range of participants in the MMSE was from 24/30 to the ceiling score (30/30) with a total of 15 participants that did not reach the ceiling level. The study is part of the European Union's Horizon, 2020 research and innovation program and was approved by the TNO Institutional Review Board.

\subsection{Stimuli and Materials}

During the experiment, the stimuli were projected (InFocus DepthQ Projector; resolution: $800 \times 600$ pixels, $120 \mathrm{~Hz}$ ) onto a $117.9 \times 89.5 \mathrm{~cm}$ back-projection screen (Techplex 150, acrylic rear projection screen) that was tilted backward by $30^{\circ}$. At the beginning of each trial, participants started with their finger on a home position, which was a green disc with a diameter of four centimeters situated 30 centimeters below the center of the screen (i.e., at coordinates $(0,-30)$ in $\mathrm{cm}$ from the center of the screen; see Fig. 1 for an overview of the stimulus lay-out). After a random time between 600 and $1200 \mathrm{~ms}$ a target (a black disc with a diameter of six centimeters) appeared on the screen. The target started $20 \mathrm{~cm}$ above the center of the screen $(0,20)$ and moved towards the bottom of the screen with a vertical velocity of $50 \mathrm{~cm} / \mathrm{s}$ and one of five different horizontal velocities $(-24,-12,0,12$ or $24 \mathrm{~cm} / \mathrm{s})$. The target was visible for $150 \mathrm{~ms}$ and then disappeared. For the five different target motion directions, the disappearing points relative to the center of the screen $(0,0)$ were: $(-3.6,12.5),(-1.8,12.5),(0,12.5),(1.8,12.5)$ and $(3.6,12.5)$. The targets and the home position were presented on a background of white and blue squares that formed a checkerboard that filled the whole screen. The squares' sides were five centimeters long. For the targets moving in an oblique direction (horizontal velocities: $-24,-12,12$ or $24 \mathrm{~cm} / \mathrm{s}$ ), the background started to move at $12 \mathrm{~cm} / \mathrm{s}$ to the right or the left as soon as the target appeared. For targets going straight downwards (horizontal velocity: $0 \mathrm{~cm} / \mathrm{s}$ ), the background 

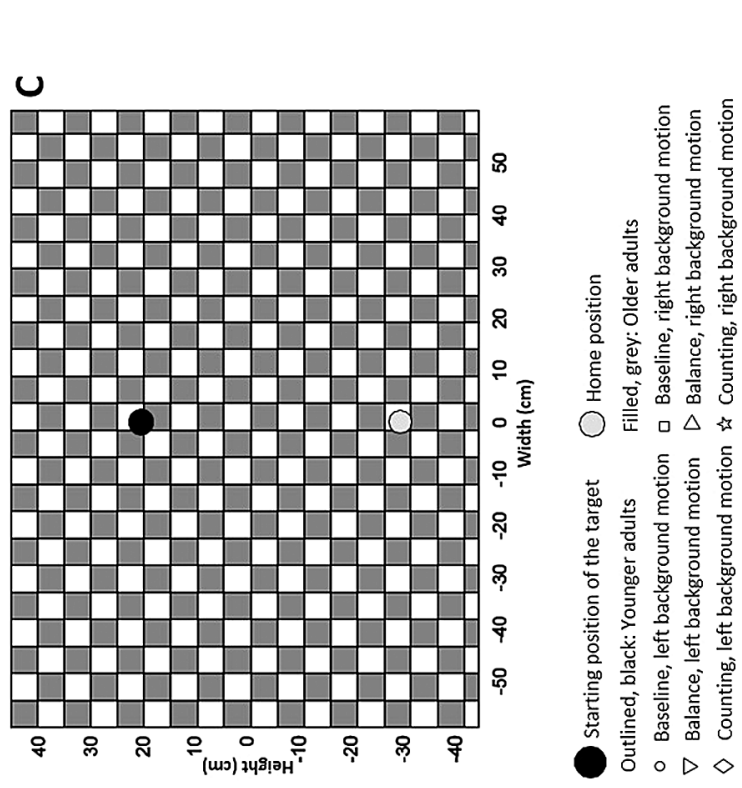

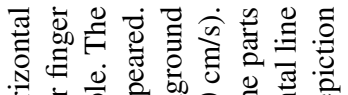

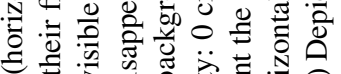

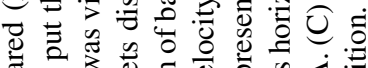

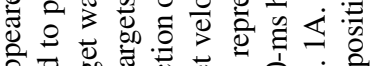

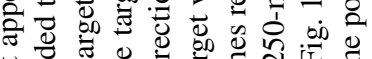

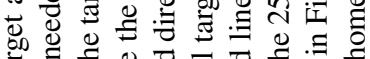

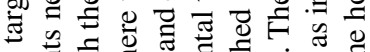
o

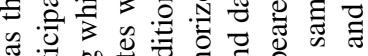
空

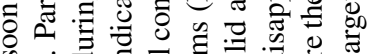

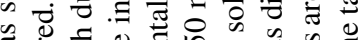

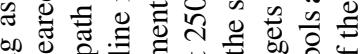
年

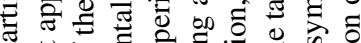
के

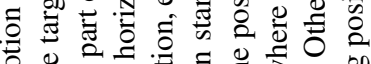
월

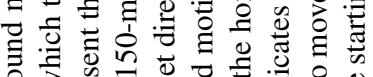

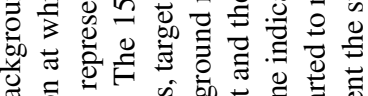

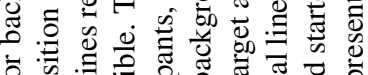

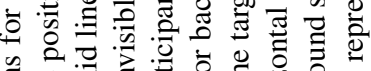

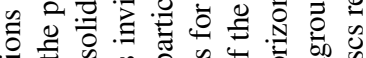

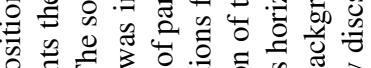
过 on

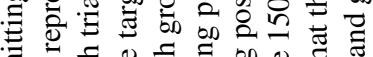

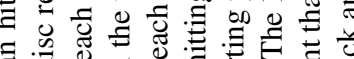
踏

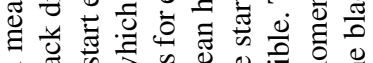

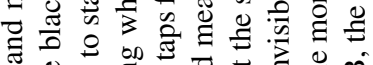

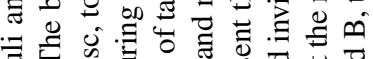

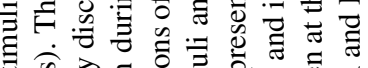

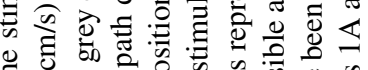
ए人

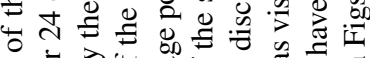

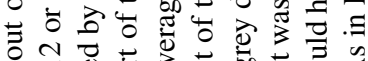

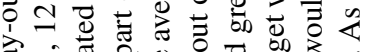

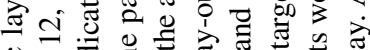

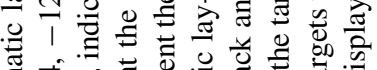

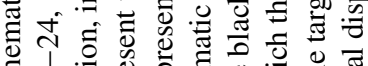

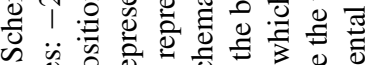

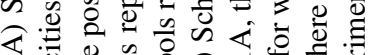

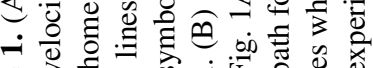

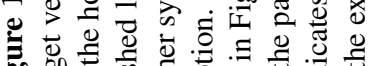

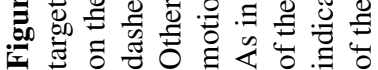


started to move with a speed of $12 \mathrm{~cm} / \mathrm{s}$ to the right or the left, $250 \mathrm{~ms}$ after the target had appeared, which was $100 \mathrm{~ms}$ after the target had disappeared. An infrared emitting diode (IRED) was placed on the participant's right index finger and was tracked at $500 \mathrm{~Hz}$ by an Optotrak (Northern Digital, Waterloo, ON, Canada). Taps were detected using a threshold deceleration of $50 \mathrm{~m} / \mathrm{s}^{2}$, which has proven to be a very reliable method (Brenner and Smeets, 2015).

Auditory stimuli were presented to the participants by a computer situated to their right. The computer presented sequences of low and high tones ( $250 \mathrm{~Hz}$ for $100 \mathrm{~ms}$ for $60 \%$ of the tones; $1 \mathrm{kHz}$ for $500 \mathrm{~ms}$ for the remaining $40 \%$ ). The intervals between the tones were drawn from a uniform distribution from 2 to $6 \mathrm{~s}$. These stimuli were always presented, but participants only had to pay attention to them in the condition in which they had to count the tones. The block of foam on which participants had to stand in the balance condition and in one of the pretests had a length and width of $40 \mathrm{~cm}$, a height with no load of $15 \mathrm{~cm}$, a height of about $10 \mathrm{~cm}$ when compressed by the weight of a participant, and a density of $35 \mathrm{~kg} / \mathrm{m}^{3}$.

\subsection{Design}

The interception task in the baseline condition was similar to the task in Brouwer et al. (2003). Participants were free to move their head, but they were either sitting on a high chair or standing on a block of foam. The chair and the foam were placed in a position from which the participant could easily reach all relevant parts of the screen. Consequently, the participants' eyes were at a distance of about $60 \mathrm{~cm}$ from the screen (so $1 \mathrm{~cm}$ is about one degree of visual angle). They had to hit the moving virtual targets (that had disappeared after being visible for $150 \mathrm{~ms}$ ) as quickly and as accurately as possible with their right index finger. Participants received feedback about their performance after hitting the screen. If they hit the target, the target reappeared and remained static at the position that it had reached at the time of the hit. If they missed, the target reappeared and moved in the opposite direction of the error. Thus, for instance, if the participant hit to the right and below the real position of the target, the target would reappear and move upwards and to the left. Such feedback might help participants learn not to rely on the background. Beside the baseline condition, there were two other conditions.

In the balance condition participants were standing on a compliant surface (the above-mentioned block of foam) rather than sitting. This makes it more challenging to maintain one's posture while making the required hitting movement. In the counting condition, participants were sitting but had to remember the number of high and low tones that they heard. These two secondary tasks are expected to stress different processes of integration; one is 
mainly cognitive (counting) and one is more proprioceptive (balance). We examined whether the additional challenges affect older participants more than younger participants.

\subsection{Protocol}

Participants first performed four standardized clinical tests that are currently used to gauge mental and physical fitness of older adults. Participants were screened for cognitive impairments with the MMSE (Dick et al., 1984), for sensory integration and balance deficits with the m-CTSIB (Horn and Scherer, 2015; Shumway-Cook and Horak, 1986), for lower limb physical functioning dysfunctions with the SPPB (Guralnik et al., 1994; Pavasini et al., 2016) and for difficulties in performing instrumental activities of daily living with the Instrumental Activities of Daily Living scale (Lawton and Brody, 1969). The pretests were done in the same order for all participants, with the physically demanding tasks done before the non-physically demanding ones to avoid fatigue effects carrying over to the interception task. The order was the following: m-CTSIB, SPPB, MMSE and instrumental activities of daily living questionnaire. A regular chair was used for chair stand tests, the abovementioned block of foam to perturb balance and a $4 \mathrm{~m}$ long walking course (indicated by pieces of tape on the floor) to assess gait speed in the SPPB.

Before doing the interception task, participants were provided with written instructions regarding the entire task and procedures. The task was to hit the targets with their finger as quickly and accurately as possible. After reading the instructions, the examiner showed the participants how to do the interception task. Participants then performed a practice session while sitting in front of the screen. The practice session consisted of ten trials (two trials for each of the five possible directions of target motion) with the target remaining visible during the entire trial and an unlimited number of randomized trials with the target disappearing after $150 \mathrm{~ms}$. They were asked to practice until they felt comfortable with the task. Once they indicated that they had practiced enough the actual experiment was done. The examiner ensured that participants were able to hear the tones before starting the experiment. The computer delivering the tones was moved closer for participants having trouble to hear the tones (two participants). The three conditions (baseline, balance, counting) were presented in random order. Each condition contained 95 trials: five additional practice trials without any background motion (one trial per direction of target motion, presented in random order) and 90 experimental trials. In the 90 experimental trials, the background moved to the left in 45 trials (nine trials per direction of target motion) and the background moved to the right in the other 45 (nine trials per direction of target motion). The three blocks were separated by short breaks during which participants read the instructions for the following condition. Participants were also allowed to take breaks at any time 


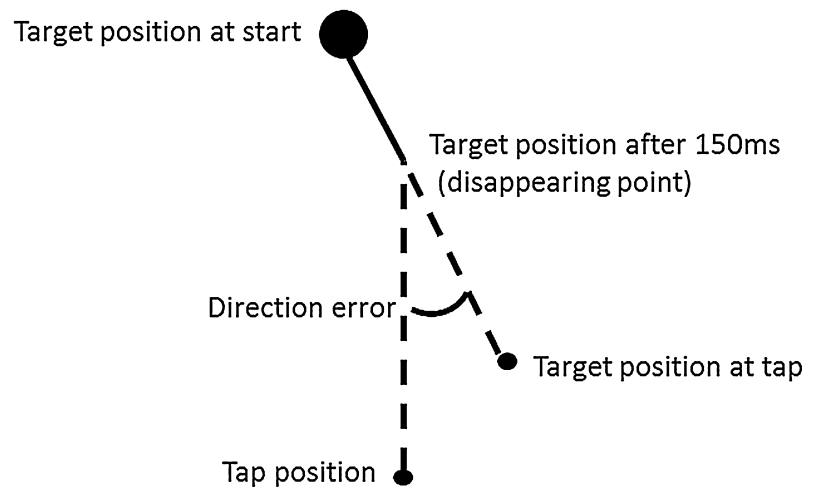

Figure 2. Definition of the direction error. The direction error is the angle in degrees between the direction of motion of the target (the line between the target position at the start and the target position at the time of hitting) and a line between the position of the target after $150 \mathrm{~ms}$ presentation time (i.e., disappearing point) and the hit position. When the hit position was to the left of the target position, as in the example here, the direction error was considered to be negative.

during the experiment. At the end of the counting block, participants had to report the number of high and low tones they had heard during the block. We used these values to ensure that they were doing the secondary task properly.

\subsection{Data Analysis}

Several dependent variables were extracted from the Optotrak data. In the data analysis, younger adults were compared to older adults; left background motion was compared to right background motion; and the balance and counting conditions were compared to the baseline condition.

\subsubsection{Direction Error}

The direction error is the angle in degrees between the direction in which the target was actually moving and the direction in which we infer that the participant considered it to be moving. The latter direction was judged from the position of the target after $150 \mathrm{~ms}$, when it disappeared, and the position that was tapped (see Fig. 2). A tapping error to the left (as shown in the example given in Fig. 2) was considered to be negative. We determined the mean direction error for each participant, experimental condition and direction of background motion. The direction error is our main dependent variable.

\subsubsection{Standard Deviation of Direction Error}

To examine whether there were any differences in performance variability between the age groups and conditions we also examined the standard deviations of the direction errors. To avoid considering biases that depend on the direction of target motion as additional variability, we determined the standard deviation for each subject, condition, direction of background motion and direction of 
target motion (horizontal velocities of $-24,-12,12$ and $24 \mathrm{~cm} / \mathrm{s}$ ), and then averaged the standard deviations across the four directions of target motion.

\subsubsection{Hit, Miss and 'No Tap' Trials}

As an additional overall measure of performance we determined the number of hits and misses. Trials were considered to be hits if the relevant part of the participant's finger hit the screen within $3 \mathrm{~cm}$ of the center of the target. They were considered to be misses if the participant failed to hit the target. In 'no tap' trials the screen was either not hit by the participants' finger at all, was tapped after the target left the screen (more than $1100 \mathrm{~ms}$ after it disappeared), or was not tapped hard enough.

\subsubsection{Average Time to Tap, Reaction Time and Movement Time}

The reaction time is the time between the presentation of a stimulus (target appearance) and the moment we were certain that the finger was moving (speed threshold of $0.3 \mathrm{~m} / \mathrm{s}$ ). The movement time is the time between this moment and the moment that the finger hit the screen. The time to tap is the sum of these two times: the time between the presentation of a stimulus and when the finger hit the screen.

\subsection{Statistical Analysis}

We considered effects of age group (older adults and younger adults), background motion (left and right) and condition (baseline, counting and balance). For our main question we used a three-way ANOVA to evaluate how the direction error depends on age group, experimental condition and background motion. We expected to find a main effect of background motion, but were interested in determining whether there was a significant interaction between age group and background motion (possibly indicating that the background motion had a stronger effect on older adults), whether there was a significant interaction between condition and background motion (indicating that the secondary tasks influence the effect of the background motion), and whether there was a three-way interaction (possibly indicating that older adults are more susceptible to the background motion under certain conditions). Some additional tests were conducted to evaluate the other variables.

The normality of distribution of the residuals and the equality of the variances between the groups and conditions were tested with the MATLAB functions qqplot and vartest2, respectively. If these parameters appeared to be normally distributed we used ANOVAs to examine main effects and interactions and $t$-tests to evaluate specific comparisons (such as whether a significant effect of condition was due to a difference between the baseline and the counting condition or to a difference between the baseline and the balance condition). If the residuals were not normally distributed or the variances were not equal, non-parametric tests were used: the Mann-Whitney $U$ test (unpaired samples, 
two tailed $p$-value) for differences due to age, and the Wilcoxon signed-rank test (paired samples) for differences between conditions. Bonferroni corrections were used to adjust $p$-values when doing multiple comparisons. All significant effects $(p<0.05)$ are reported. Significant differences are represented with asterisks in the figures; *: $p<0.05 ; * *: p<0.01 ; * *: p<0.001$.

\section{Results}

\subsection{Participants}

No participant was excluded from the study because of a low MMSE score or failing on any of the other clinical tests. Three participants, one younger adult and two older adults, were excluded from the analysis because they had not counted the tones during the counting condition. Three older adults were excluded from the analysis because their performance was very different from the others. While the other participants' direction errors were all between -10 and +15 degrees, these participants' mean direction errors were up to -25 and +70 degrees, although they did not score differently from the other older adults in the pretests. Looking at the tap positions per participant revealed that these three older adults tended to tap where the target disappeared rather than where it would be at the time of the tap. We interpret this as them not having understood the task properly.

\subsection{Counting Tones}

Participants were asked to report the number of tones at the end of the counting block. The reported number provided the examiner with an impression of how well the participant had adhered to the secondary task. The task must have been challenging, because almost all of the subjects gave a slightly incorrect answer. Further evidence that the counting task was challenging is that the number of no taps was larger for the counting task condition than for the other conditions (see later in the results).

\subsection{Direction Errors}

Figure 3 shows direction errors in degrees. The expected effect of the direction of background motion is clearly visible: participants have a more positive direction error when the background moves to the left than when the background moves to the right $[F(1,216)=39.71, p<0.001]$. This effect is stronger for older adults than younger adults [interaction between age and background motion: $F(1,216)=9.63, p=0.002$ ]. Increasing the difficulty of maintaining balance (standing on foam rather than sitting) and having to count tones while performing the task had no systematic effect on the direction error: 


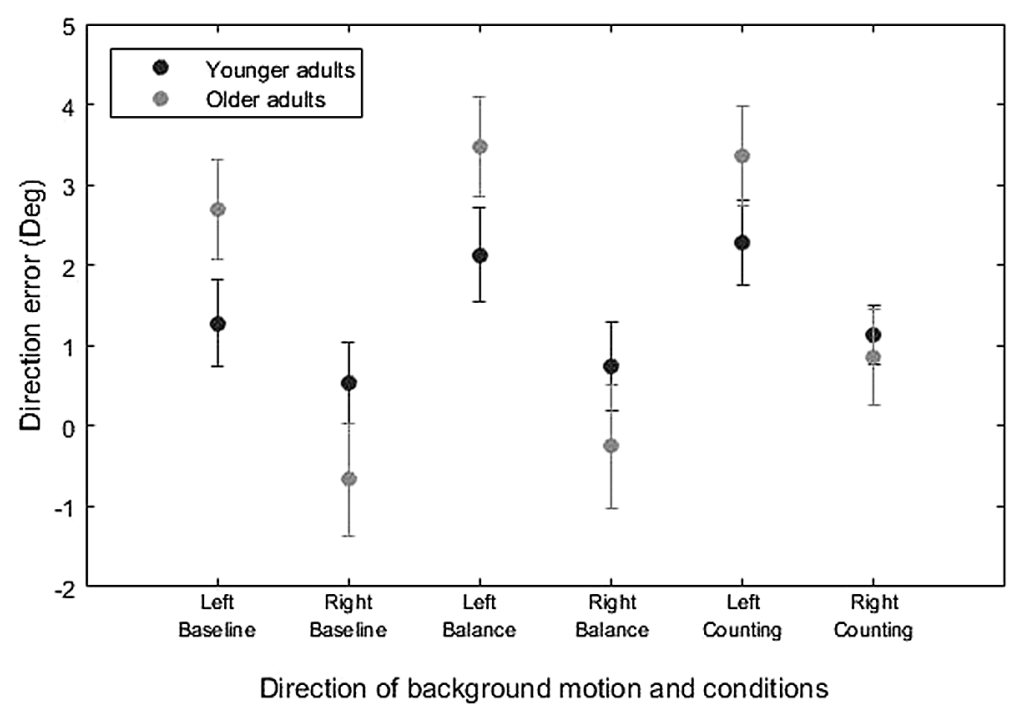

Figure 3. Average direction error in degrees according to age group, experimental condition (baseline, balance and counting) and background direction of motion (left or right) merged between the different directions of the target's motion (horizontal velocities: $-24,-12,12$ and $24 \mathrm{~cm} / \mathrm{s}$ ). Error bars represent the standard error of the mean between subjects. Especially, older subjects hit more to the right when the background moved to the left and more to the left when the background moved to the right. This is consistent with an illusory direction of motion caused by the moving background.

there was no significant interaction between condition and background motion $[F(2,216)=0.39, p=0.68]$ or between condition, age and background motion $[F(2,216)=0.32, p=0.72]$.

\subsection{General Performance Measures}

\subsubsection{Standard Deviation of Direction Error}

The standard deviations of the direction errors were significantly larger for the older adults in all experimental conditions and both for leftward and rightward background motion (Mann-Whitney $U$ test: all $p<0.01$ ), indicating that the older participants' performance was more variable than that of the younger participants. The standard deviations of direction error did not differ significantly between the experimental conditions for either the leftward or rightward background motion for either group (Wilcoxon signed-rank tests: $p>0.05$ ), except in the older adult group where the standard deviation was significantly larger in the counting condition than in the baseline condition for leftward background motion $(Z=-2.95, p=0.025)$. 


\subsubsection{Tapping Positions}

Figure 1 shows the average position of the tap for each group of participants (young, old), experimental condition (baseline, balance, counting), background motion (left or right) and background timing (starting to move immediately or after $250 \mathrm{~ms}$ ). The figure shows that older adults move less far from the starting point and tap closer to the center of the screen than younger adults. If this undershooting bias results from the older participants moving less far than they intended to move, independently of any judgment of target motion, we will even be underestimating the influence that the background had on the older participants' responses, because any direction differences are reduced by such a bias (for our starting point).

\subsubsection{Percentage of Hits and Number of 'No Tap' Trials}

Not surprisingly, considering the tendency to undershoot the target's path (Fig. 1), the larger systematic errors introduced by the background motion (Fig. 3) and the larger variability (Fig. 4), older participants hit fewer targets than younger participants (Fig. 5). A significant effect of age [two-way ANOVA: $F(1,108)=37.1, p<0.001]$ was accompanied by a significant effect of condition $[F(2,108)=3.46, p=0.035]$, but no interaction between

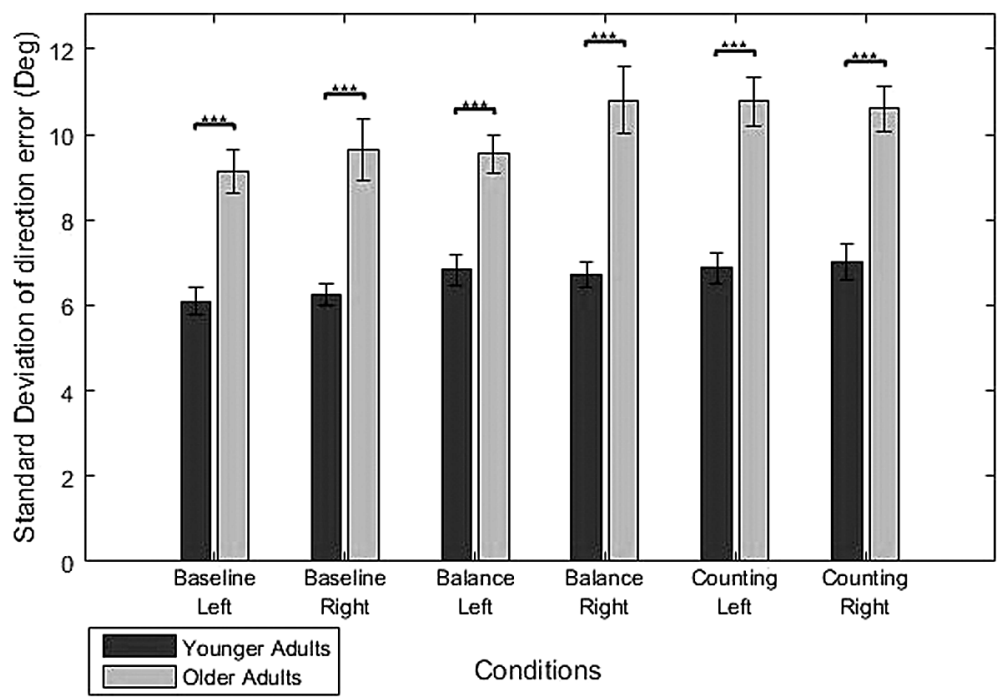

Figure 4. Standard deviation of direction error in degrees according to age, experimental condition (baseline, balance and counting) and background direction of motion (left or right) averaged between the different directions of the target's motion (horizontal velocities: $-24,-12,12$ and $24 \mathrm{~cm} / \mathrm{s}$ ). Error bars represent the standard error of the mean between subjects. Asterisks represent significant differences: $*: p \leqslant 0.05, * *: p \leqslant 0.01, * * *: p \leqslant 0.001$. The standard deviations of older adults are larger compared to younger adults showing that older adults are more variable than younger adults in their direction errors. 


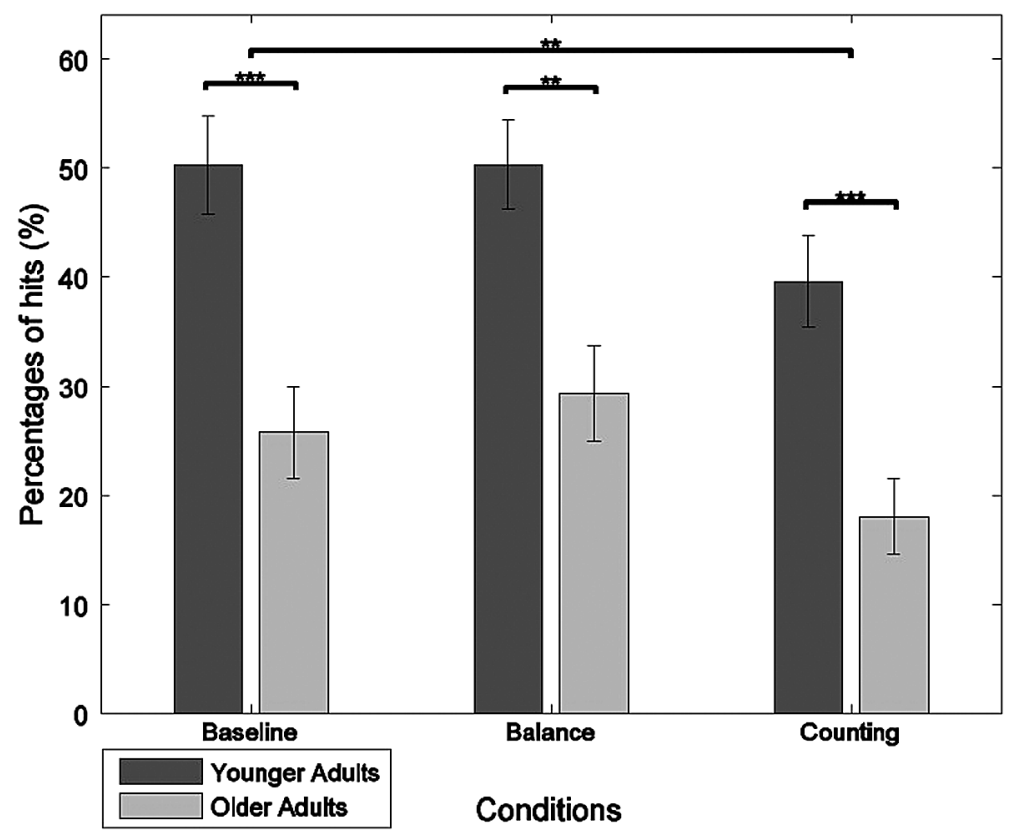

Figure 5. Percentage of hits according to age and experimental condition (baseline, balance and counting) merged between the different directions of the target's motion (horizontal velocities: $-24,-12,12$ and $24 \mathrm{~cm} / \mathrm{s}$ ). Error bars represent the standard error of the mean between subjects. Asterisks represent significant differences: *: $p \leqslant 0.05, * *: p \leqslant 0.01, * * *: p \leqslant 0.001$. Older participants hit fewer targets than younger participants. The percentage of hits was lower in the counting condition than in the baseline condition for both age groups.

age and condition $[F(2,108)=0.34, p=0.7]$. The effect of condition was due to the percentage of hits being lower in the counting condition than in the baseline condition $[t(38)=3.3, p=0.002]$. There was no difference between the baseline and the balance conditions [ $t(38)=-0.94, p=0.36]$. Older adults also had a higher number of 'no tap' trials than younger adults [this was significant in the balance and counting conditions (Mann-Whitney $U$ test: both $p<0.001$ ) but not in the baseline condition; $p=0.11$ ].

\subsubsection{Average Times}

The average time to tap depended on the condition $[F(2,113)=13.11$, $p<0.001]$ but there was no significant effect of age $[F(1,113)=2.69$, $p=0.104]$ or significant interaction between age and condition $[F(2,113)=$ $0.2, p=0.82$ ] (Fig. 6). The average time to tap was shorter in the balance condition $[t(37)=3.35, p=0.002]$ and longer in the counting condition $[t(37)=-6.17, p<0.001]$ than in the baseline condition. The reaction time alone showed a similar pattern of results. The movement time depended on age $[F(1,113)=6.53, p=0.012]$ as well as condition $[F(2,113)=5.97$, 


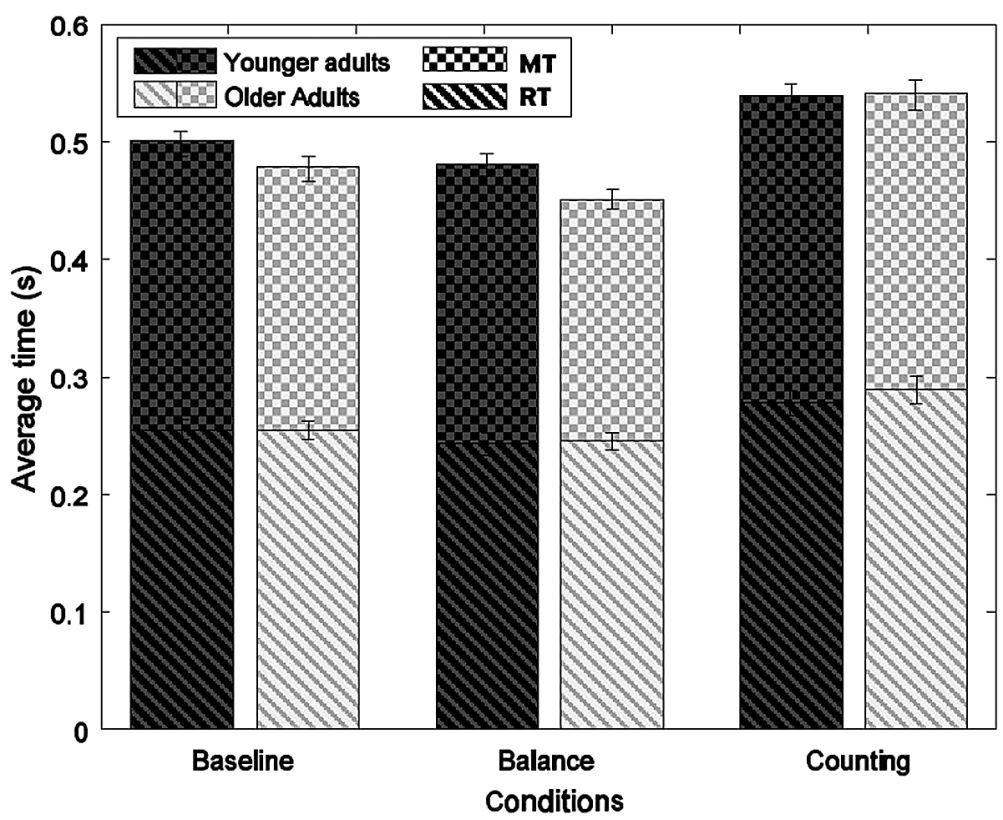

Figure 6. Average time to tap (s) split into reaction time (RT) and movement time (MT) according to age and experimental condition (baseline, balance and counting) merged between the different directions of the target's motion (horizontal velocities: $-24,-12,12$ and $24 \mathrm{~cm} / \mathrm{s}$ ). Error bars represent the standard error of the mean between subjects. The average time to tap was shorter in the balance condition and longer in the counting condition than in the baseline condition. The reaction time and movement time showed a similar pattern of results between the conditions. The movement time was shorter for older adults.

$p=0.004]$. There was no interaction between age and condition $[F(2,113)=$ $0.61, p=0.5]$. The movement time was shorter for older adults, and it too was shorter in the balance condition and longer in the counting condition than in the baseline condition.

\subsection{Late Background Motion}

\subsubsection{Direction Errors}

Figure 7 shows the direction errors in degrees for the condition in which the target moved straight downward and the background started to move $250 \mathrm{~ms}$ after the target appeared, i.e., $100 \mathrm{~ms}$ after it had disappeared. The expected effect of the direction of background motion is clearly visible: participants have a more positive direction error when the background moves to the right and more negative errors when the background moves to the left (Wilcoxon signed-rank test: all $p<0.01$ except for the comparison of left and right background motion for the balance condition in older adults, where $p=0.053$ ). This effect is clearly not stronger for older adults than for younger adults, but the converse is also not significant (Mann-Whitney $U$ test: $p>0.05$ 


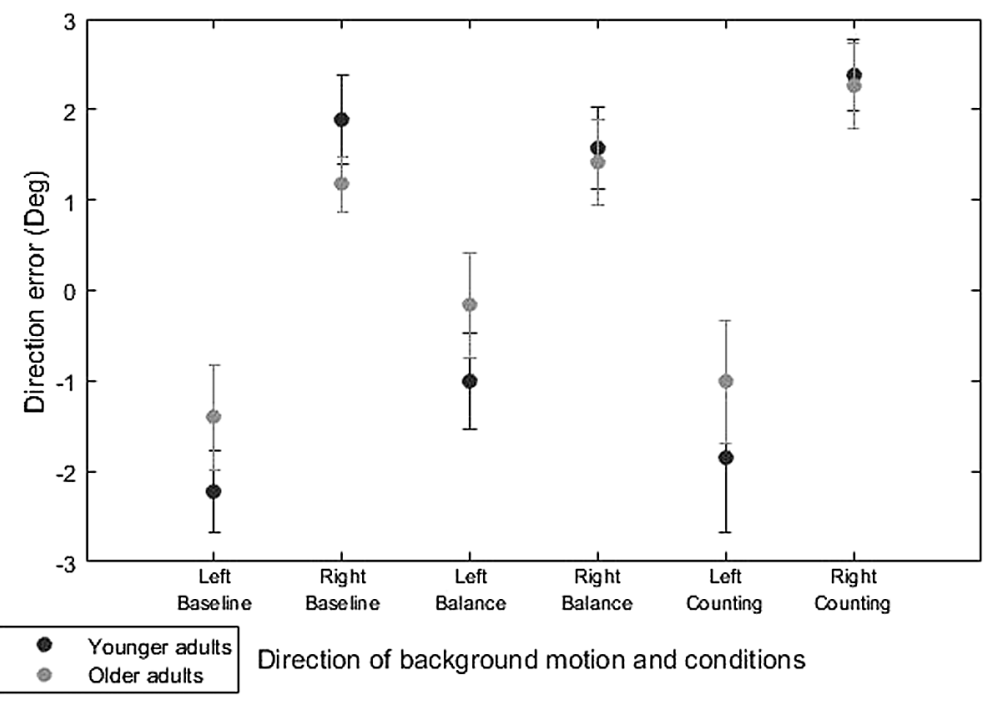

Figure 7. Direction error in degrees according to age group, experimental condition (baseline, balance and counting) and background direction of motion (left or right) for targets moving vertically (horizontal velocity: $0 \mathrm{~cm} / \mathrm{s}$ ) and for a background motion starting at $100 \mathrm{~ms}$ after the target had disappeared. Error bars represent the standard error of the mean between subjects. Subjects hit too far to the right when the background moved to the right and too far to the left when the background moved to the left, i.e. an effect opposite of the illusory direction of motion effect. Here there is no difference between age groups. MT: Movement time; RT: reaction time.

for all the three conditions). Increasing the difficulty of maintaining balance (standing on foam rather than sitting) and having to count tones while performing the task had no systematic effect on the direction error; no significant differences were found for older adults nor for younger adults between the balance and baseline conditions and between the counting and baseline conditions for left- and right-moving backgrounds (Wilcoxon signed-rank test: all $p>0.05)$.

\subsubsection{Hand Velocity}

Figure 8 shows the timing of the response of the hand to abrupt background motion $250 \mathrm{~ms}$ after the target appeared. This figure shows that the initial response to the background's motion is also no larger for older adults than younger adults. Older adults might have slightly longer latencies. There is no evident difference between the responses for the different conditions.

\subsection{Pretests Results}

All the participants were at ceiling level for the m-CTSIB (score 120/120). The score range of participants in the SPPB was from 10/12 (one participant) to the ceiling score (12/12). Seven participants had a score of $11 / 12$. The differences between participants in this test were due to the chair stand test. All 

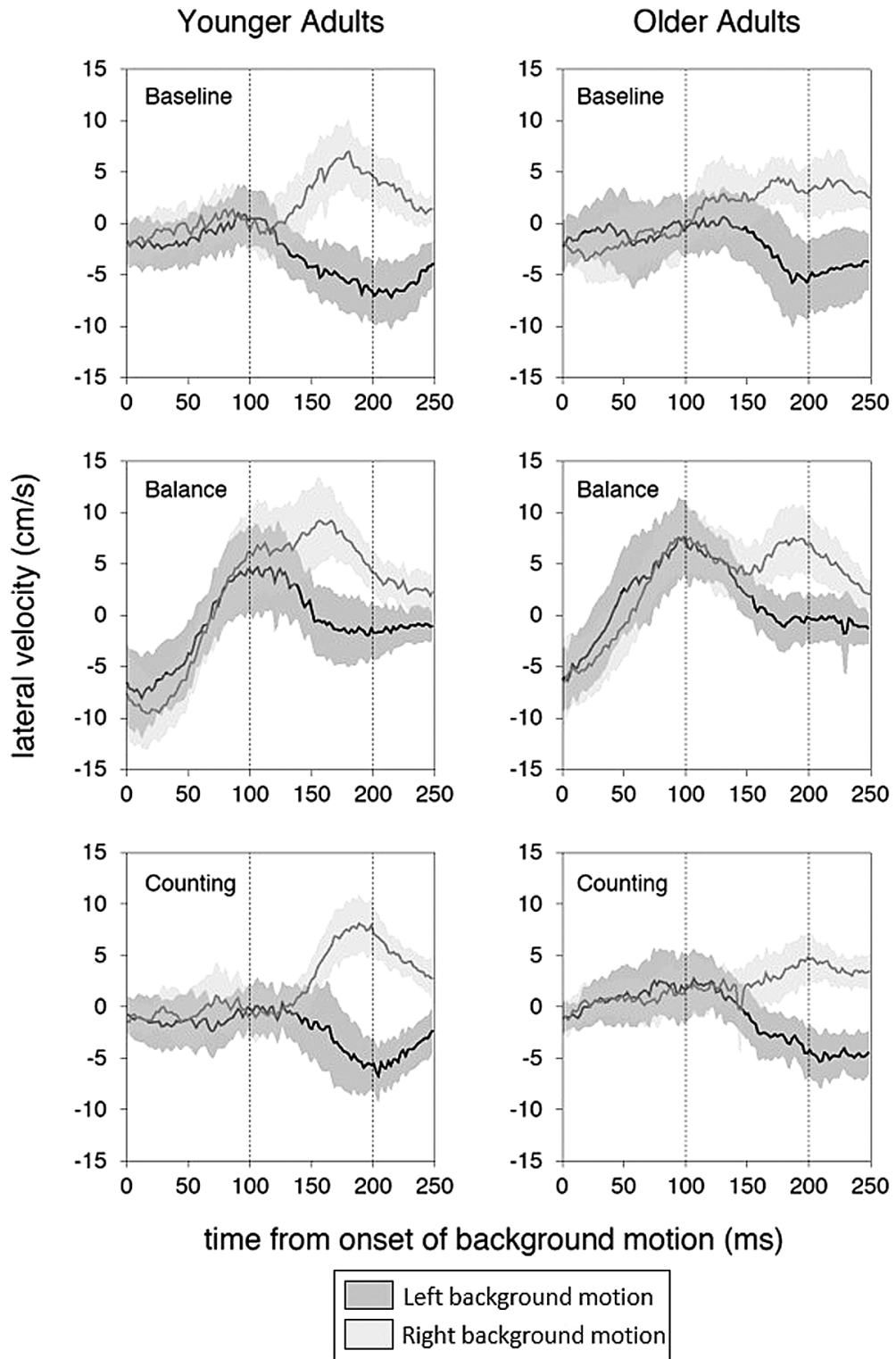

Figure 8. Average lateral hand velocities of the subjects $(\mathrm{cm} / \mathrm{s})$ relative to the time of background motion onset (ms) for younger and older adults. Older adults' responses to the late background motion are not larger than the ones of younger adults.

the participants were at ceiling level in the instrumental activities of daily living questionnaire (score 8/8) meaning that they had no problems performing the activities of daily life. 


\section{Discussion}

\subsection{Effects of Age}

The present study aimed at investigating whether older adults were affected more by task-irrelevant background motion in an interception task than young adults, and whether this difference became more prominent under more demanding conditions. Older adults were indeed more susceptible to the background motion. Their overall performance was also poorer: their direction errors were more variable, they successfully hit the target in fewer trials and they had a higher number of 'no tap' trials. Older adults responded as quickly as younger adults, and moved slightly faster. Moving faster might partly explain older adults' larger variability of direction errors. However, the fact that older adults were not faster than younger adults in the counting condition while they had the same difference in variability as for the other conditions, suggests that a speed accuracy trade-off can only play a rather minor role. The age-related differences in the effects of background motion on the interception task are consistent with the results of previous studies (for a review see de Dieuleveult et al., 2017) and particularly with the results of Berard and collaborators (2012) who found a similar increase in sensitivity to inappropriate optic flow. However, in our experiment, both young adults and older adults were affected significantly by the background motion, while only older adults were affected in Berard's experiment.

In the present study we focused on the use of information that was clearly inaccurate as indicated by the feedback. This intuitively led to the expectation that participants should have avoided using any information that is affected by the background motion. The effect of background motion presumably arises from the way in which information for judging the target's direction of motion is acquired. In principle, motion of the target in space could be acquired by combining motion of the target's image on the retina with information about changes in the eye's orientation in the head and changes in the orientation of the head (Nakayama, 1985; Schweigart et al., 2003). However, one could also rely on the surrounding being static, as it usually is, and also use the relative motion of the target's retinal image and that of its surrounding to estimate the target's motion in space. Using the relative motion in this way, rather than only using information that relies heavily on extra-retinal information, means that one will be fooled if the background is moving (Brenner and van den Berg, 1994), because background motion will be interpreted as optic flow due to our own motion, but it will increase the precision whenever the background is not moving. With age, the decline in the resolution of information from the eye muscles and from the vestibular system might be more severe than the decline in the visual resolution. If so, giving more weight to relative motion when 
one is older might be optimal in terms of minimizing the variability in performance (Ernst and Banks, 2002; Van Beers et al., 1999). Thus, the stronger influence of the background in older adults may be due to a decline in the resolution of proprioception and of the vestibular system, rather than to a deficit in combining sensory information. One might be surprised that even our younger participants continued to rely on the background being static despite the feedback. However, in a study in which providing feedback certainly shifted the weights given to cues (Van Beers et al., 2011), the cue that was inconsistent with the feedback was still not ignored altogether.

Figures 7 and 8 show that older adults are not always more susceptible to the effects of background motion than are younger adults, which supports the idea that the difference in performance is specific to judgments of the target's motion. Still, given earlier studies that support the idea of older adults generally having trouble down-regulating non-relevant or misleading information (Berard et al., 2012; de Dieuleveult et al., 2017; Eikema et al., 2014; McGovern et al., 2014; Teasdale et al., 1991), we do not think that the effects of aging are specific to judging the direction of target motion.

\subsection{Effects of Additional Tasks}

The two secondary tasks had no effect on the direction error or the variability of direction error in either age group. This is not in accordance with our hypothesis and previously reported effects (Bisson et al., 2014; de Dieuleveult et al., 2017; Mahboobin et al., 2007; Redfern et al., 2001, 2009). The time that it took to make the movements was shorter in the balance condition than in the baseline condition. Perhaps the movement was easier to make when standing, or perhaps there was more reason to be fast when one's posture was less stable. It took longer to make the movements in the counting condition, in which the percentage of hits was smaller and there were more 'no tap' trials. That the counting condition was more difficult than the two other conditions is supported by the observation that in this condition some older participants tended to tap the home button instead of keeping their finger on it, even after the experimenter remarked about this. The older participants may have been unduly affected by this task because we did not systematically adjust the volume to the subjects' hearing. Although the volume was quite high, some people reported having difficulties hearing the sounds, in which case we moved the computer that generated the sounds closer to them. In sum, our results were not indicative of a (resource-dependent) mechanism in the older participants that could help counteract the effect of background motion.

\subsection{Correlations With Clinical Measures}

This study is a first step towards using the influence that background motion has on manual interception as an indicator of problems or upcoming problems 
in activities of daily living. All our participants turned out to have very high scores in the pretests, so at present we cannot look into correlations between performance on this task and problems in activities of daily living. This is partly due to the way we selected our participants. First of all, most of them were in their sixties, so they were quite young older adults. Secondly, in order to perform the experiment, participants needed to be able to come to the testing location (VU, Amsterdam), which required a good level of mobility. Considering that we did find an overall effect of aging on the susceptibility to background motion in the present study, future research will use a portable setup to be able to include a group of older adults with difficulties in the clinical measures in addition to healthy, mobile older adults to test for correlations. A reliable correlation opens the way to develop a toolkit for the early detection of problems in the older adults population.

\subsection{Limitations}

One observation that has to be taken into account when interpreting some of the differences in performance is that most of the older adults tapped the screen with their hand completely open, while all of the younger adults tapped with their fist clenched and only their index finger extended towards the screen. This might be because older adults are less used to interacting with touch screens (even though the screen used in the present study was not a touch screen, the interaction is the same). Most of the older adults initially tapped really hard on the screen, and we had to tell them that they did not need to tap that hard (to avoid injuries and fatigue). Changing the way they tapped may have increased the difficulty of the task for older adults compared to younger adults. Moreover, some of the younger participants were students of human movement science, who may be particularly good at performing this kind of task. Thus both groups of participants may not be completely representative of the age group, with a possible bias towards good performance. However, while these differences in strategy between younger and older adults may have affected the main effect of participant group, they cannot explain the differences in background motion effects between the groups.

\section{Funding}

This research was performed in the context of the PACE (Perception and Action in Complex Environments) project, which is funded from the European Union's Horizon, 2020 research and innovation program under the Marie Sklodwska-Curie grant agreement No 642961.

\section{Conflicts of Interest}

The authors declare no conflict of interest relating to this study. 


\section{References}

Atchley, P. and Andersen, G. J. (1998). The effect of age, retinal eccentricity, and speed on the detection of optic flow components, Psychol. Aging 13, 297-308.

Bennett, P. J., Sekuler, R. and Sekuler, A. B. (2007). The effects of aging on motion detection and direction identification, Vis. Res. 47, 799-809.

Berard, J., Fung, J. and Lamontagne, A. (2012). Impact of aging on visual reweighting during locomotion, Clin Neurophysiol. 123, 1422-1428.

Bisson, E. J., Lajoie, Y. and Bilodeau, M. (2014). The influence of age and surface compliance on changes in postural control and attention due to ankle neuromuscular fatigue, Exp. Brain Res. 232, 837-845.

Brenner, E. and Smeets, J. B. J. (1997). Fast responses of the human hand to changes in target position, J. Mot. Behav. 29, 297-310.

Brenner, E. and Smeets, J. B. J. (2015). How moving backgrounds influence interception, PloS One 10, e119903. DOI:10.1371/journal.pone.0119903.

Brenner, E. and Van den Berg, A. V. (1994). Judging object velocity during smooth pursuit eye movements, Exp. Brain Res. 99, 316-324.

Brenner, E. and Van den Berg, A. V. (1996). The special role of distant structures in perceived object velocity, Vis. Res. 36, 3805-3814.

Brouwer, A.-M., Brenner, E. and Smeets, J. B. J. (2002). Hitting moving objects: is target speed used in guiding the hand? Exp. Brain Res. 143, 198-211.

Brouwer, A.-M., Middelburg, T., Smeets, J. B. and Brenner, E. (2003). Hitting moving targets: a dissociation between the use of the target's speed and direction of motion, Exp. Brain Res. 152, 368-375.

Christensen, H., Mackinnon, A., Jorm, A. F., Henderson, A. S., Scott, L. R. and Korten, A. E. (1994). Age differences and interindividual variation in cognition in community-dwelling elderly, Psychol. Aging 9, 381-390.

Conlon, E. G., Power, G. F., Hine, T. J. and Rahaley, N. (2017). The impact of older age and sex on motion discrimination, Exp. Aging Res. 43, 55-79.

Cruz-Jentoft, A. J., Baeyens, J. P., Bauer, J. M., Boirie, Y., Cederholm, T., Landi, F., Martin, F. C., Michel, J. P., Rolland, Y., Schneider, S. M., Topinková, E., Vandewoude, M., Zamboni, M., European Working Group on Sarcopenia in Older People (2010). Sarcopenia: European consensus on definition and diagnosis: report of the European Working Group on Sarcopenia in Older People, Age Aging 39, 412-423.

de Dieuleveult, A. L., Siemonsma, P. C., van Erp, J. B. and Brouwer, A. M. (2017). Effects of aging in multisensory integration: a systematic review, Front. Aging Neurosci. 9, 80. DOI:10. 3389/fnagi.2017.00080.

DeLoss, D. J., Pierce, R. S. and Andersen, G. J. (2013). Multisensory integration, aging, and the sound-induced flash illusion, Psychol. Aging 28, 802-812.

Deshpande, N. and Zhang, F. (2014). Trunk, head, and step characteristics during normal and narrow-based walking under deteriorated sensory conditions, J. Mot. Behav. 46, 125-132.

Dick, J. P. R., Guiloff, R. J. and Stewart, A. (1984). Mini-mental state examination in neurological patients, J. Neurol. Neurosurg. Psychiat. 47, 496-499.

Diederich, A., Colonius, H. and Schomburg, A. (2008). Assessing age-related multisensory enhancement with the time-window-of-integration model, Neuropsychologia 46, 2556-2562.

Duncker, K. (1929). Über induzierte Bewegung, Psychol. Forsch. 12, 180-259. 
Eikema, D. J. A., Hatzitaki, V., Tzovaras, D. and Papaxanthis, C. (2014). Application of intermittent galvanic vestibular stimulation reveals age-related constraints in the multisensory reweighting of posture, Neurosci. Lett. 561, 112-117.

Ernst, M. O. and Banks, M. S. (2002). Humans integrate visual and haptic information in a statistically optimal fashion, Nature 415(6870), 429-433.

Glisky, E. L. (2007). Changes in cognitive function in human aging, in: Brain Aging: Models, Methods, and Mechanisms, D. R. Riddle (Ed.), pp. 3-20. CRC Press/Taylor and Francis, Boca Raton, FL, USA.

Guerreiro, M. J. S., Anguera, J. A., Mishra, J., Van Gerven, P. W. and Gazzaley, A. (2014). Ageequivalent top-down modulation during cross-modal selective attention, J. Cogn. Neurosci. 26, 2827-2839.

Guerreiro, M. J. S., Eck, J., Moerel, M., Evers, E. A. and Van Gerven, P. W. (2015). Top-down modulation of visual and auditory cortical processing in aging, Behav. Brain Res. 278, 226234.

Guralnik, J. M., Simonsick, E. M., Ferrucci, L., Glynn, R. J., Berkman, L. F., Blazer, D. G., Scherr, P. A. and Wallace, R. B. (1994). A short physical performance battery assessing lower extremity function: association with self-reported disability and prediction of mortality and nursing home admission, J. Gerontol. 49, M85-M94.

Horn, L. B. and Scherer, M. R. (2015). Measurement characteristics and clinical utility of the clinical test of sensory interaction on balance (CTSIB) and modified CTSIB in individuals with vestibular dysfunction, Arch. Phys. Med. Rehab. 96, 1747-1748.

Hugenschmidt, C. E., Peiffer, A. M., McCoy, T. P., Hayasaka, S. and Laurienti, P. J. (2009). Preservation of crossmodal selective attention in healthy aging, Exp. Brain Res. 198, 273285.

Katz, S. (1963). Studies of illness in the aged, J. Am. Med. Assoc. 185, 914.

Kavcic, V., Vaughn, W. and Duffy, C. J. (2011). Distinct visual motion processing impairments in aging and Alzheimer's disease, Vis. Res. 51, 386-395.

Lawton, M. P. and Brody, E. M. (1969). Assessment of older people: self-maintaining and instrumental activities of daily living, Gerontologist 9, 179-186.

Lowry, K. A., Vallejo, A. N. and Studenski, S. A. (2012). Successful aging as a continuum of functional independence: lessons from physical disability models of aging, Aging Dis. 3, $5-15$.

Mahboobin, A., Loughlin, P. J. and Redfern, M. S. (2007). A model-based approach to attention and sensory integration in postural control of older adults, Neurosci. Lett. 429, 147-151.

McGovern, D. P., Roudaia, E., Stapleton, J., McGinnity, T. M. and Newell, F. N. (2014). The sound-induced flash illusion reveals dissociable age-related effects in multisensory integration, Front. Aging Neurosci. 6, 250. DOI:10.3389/fnagi.2014.00250.

Nakayama, K. (1985). Biological image motion processing: a review, Vis. Res. 25, 625-660.

Newell, K. M., Vaillancourt, D. E. and Sosnoff, J. J. (2006). Handbook of the Psychology of Aging. Elsevier, Amsterdam, The Netherlands.

Norman, J. F., Ross, H. E., Hawkes, L. M. and Long, J. R. (2003). Aging and the perception of speed, Perception 32, 85-96.

Owsley, C. (2011). Aging and vision, Vis. Res. 51, 1610-1622.

Pavasini, R., Guralnik, J., Brown, J. C., di Bari, M., Cesari, M., Landi, F., Vaes, B., Legrand, D., Verghese, J., Wang, C., Stenholm, S., Ferrucci, L., Lai, J. C., Bartes, A. A., Espaulella, 
J., Ferrer, M., Lim, J. Y., Ensrud, K. E., Cawthon, P., Turusheva, A., Frolova, E., Rolland, Y., Lauwers, V., Corsonello, A., Kirk, G. D., Ferrari, R., Volpato, S. and Campo, G. (2016). Short physical performance battery and all-cause mortality: systematic review and metaanalysis, BMC Med. 14(1), 215. DOI:10.1186/s12916-016-0763-7.

Pilz, K. S., Bennett, P. J. and Sekuler, A. B. (2010). Effects of aging on biological motion discrimination, Vis. Res. 50, 211-219.

Ramkhalawansingh, R., Keshavarz, B., Haycock, B., Shahab, S. and Campos, J. L. (2017). Examining the effect of age on visual-vestibular self-motion perception using a driving paradigm, Perception 46, 566-585.

Redfern, M. S., Jennings, J. R., Martin, C. and Furman, J. M. (2001). Attention influences sensory integration for postural control in older adults, Gait Posture 14, 211-216.

Redfern, M. S., Jennings, J. R., Mendelson, D. and Nebes, R. D. (2009). Perceptual inhibition is associated with sensory integration in standing postural control among older adults, J. Gerontol. B Psychol. Sci. Soc. Sci. 64, 569-576.

Saijo, N., Murakami, I., Nishida, S. and Gomi, H. (2005). Large-field visual motion directly induces an involuntary rapid manual following response, J. Neurosci. 25, 4941-4951.

Schweigart, G., Mergner, T. and Barnes, G. (2003). Object motion perception is shaped by the motor control mechanism of ocular pursuit, Exp. Brain Res. 148, 350-365.

Shumway-Cook, A. and Horak, F. B. (1986). Assessing the influence of sensory interaction of balance. Suggestion from the field, Phys. Ther. 66, 1548-1550.

Snowden, R. J. and Kavanagh, E. (2006). Motion perception in the ageing visual system: minimum motion, motion coherence, and speed discrimination thresholds, Perception 35, 9-24.

Soechting, J. F., Engel, K. C. and Flanders, M. (2001). The Duncker illusion and eye-hand coordination, J. Neurophysiol. 85, 843-854.

Teasdale, N., Stelmach, G. E., Breunig, A. and Meeuwsen, H. J. (1991). Age differences in visual sensory integration, Exp. Brain Res. 85, 691-696.

Townsend, J., Adamo, M. and Haist, F. (2006). Changing channels: an fMRI study of aging and cross-modal attention shifts, NeuroImage 31, 1682-1692.

Trick, G. L. and Silverman, S. E. (1991). Visual sensitivity to motion: age-related changes and deficits in senile dementia of the Alzheimer type, Neurology 41, 1437-1440.

Van Beers, R. J., Sittig, A. C. and Gon, J. J. (1999). Integration of proprioceptive and visual position-information: an experimentally supported model, J. Neurophysiol. 81, 1355-1364.

Van Beers, R. J., Van Mierlo, C. M., Smeets, J. B. and Brenner, E. (2011). Reweighting visual cues by touch, J. Vis. 11, 20. DOI:10.1167/11.10.20.

Vernooij, C. A., et al. (2016). The effect of aging on muscular dynamics underlying movement patterns changes, Front. Aging Neurosci. 8, 309. DOI:10.3389/fnagi.2016.00309.

Yeh, T. T., Cinelli, M. E., Lyons, J. L. and Lee, T. D. (2015). Age-related changes in postural control to the demands of a precision task, Hum. Mov. Sci. 44, 134-142.

Zivotofsky, A. Z. (2004). The Duncker illusion: intersubject variability, brief exposure, and the role of eye movements in its generation, Invest. Opthalmol. Vis. Sci. 45, 2867-2872. 